\title{
ATIVIDADES EDUCATIVAS NA PREVENĊ̃̃ DA AIDS EM UMA REDE BÁSICA MUNICIPAL DE SAÚDE: PARTICIPAÇÃO DO ENFERMEIRO*
}

Gilson de Vasconcelos Torres**

Bertha Cruz Enders***

TORRES, G.de V.; ENDERS, B.C. Atividades educativas na prevenção da AIDS em uma rede básica municipal de saúde: participação do enfermeiro. Rev.latino-am.enfermagem, Ribeirão Preto, v. 7, n. 2, p. 71-77, abril 1999.

Estudo exploratório descritivo, com abordagem qualitativa, que objetivou caracterizar a atuação educativa dos enfermeiros que implementam o programa de prevenção e controle das DSTS/AIDS do município de Natal/RN, realizada nas unidades básicas de saúde, com 10 (dez) enfermeiras que atuavam desenvolvendo ações educativas na prevenção da AIDS. Utilizou-se um roteiro de entrevista estruturado. Para a análise dos dados utilizou-se a técnica de análise de conteúdo. Identificouse que a maioria dos enfermeiros entenderam a educação em saúde como sendo repasse de informações, o programa de prevenção da AIDS possuía uma ação insuficiente, com ausência de estrutura no serviço e omissão da SMS quanto ao apoio institucional. As atividades no programa, eram tradicionais, pouco abrangentes, esporádicas e sem recursos materiais e humanos capacitados.

UNITERMOS: enfermeiro, educação em saúde, prevenção primária da AIDS

\section{INTRODUÇÃO}

Considerando a ação educativa em saúde como um processo dinâmico e contínuo, que objetiva capacitar indivíduos e/ou grupos da comunidade para refletirem criticamente sobre as causas e problemas de saúde, tornase patente a importância da participação do enfermeiro nesse processo de ensino-aprendizagem.

Dessa forma, torna-se necessário e inadiável o envolvimento do enfermeiro em ações educativas nos serviços básicos de saúde, principalmente no que se refere à prevenção de enfermidades infecciosas, dentre elas a Síndrome de Imunodeficiência Adquirida (AIDS).

O presente estudo focaliza a participação do(a) enfermeiro(a) frente à problemática da prevenção da AIDS ao nível primário de assistência, objetivando identificar e caracterizar a atuação do mesmo nas Unidades Básicas de Saúde (UBSs) que implementam o programa de prevenção e controle da AIDS no município de Natal/RN.

Contudo, dois anos após sua implantação, o programa de controle e prevenção das DSTs/AIDS implementado no município de Natal/RN, não tem sofrido uma avaliação quanto aos seus objetivos, metas, estratégias e atuações dos profissionais. A ausência de literatura científica que se reflete na situação do programa com relação ao trabalho desenvolvido pelos profissionais na educação em saúde para a prevenção da AIDS conduziram-nos a investigar o seguinte problema de pesquisa: Qual a percepção que possuem os enfermeiros que atuam no programa de prevenção da AIDS nas UBSs sobre as ações educativas desenvolvidas na prevenção dessa enfermidade?

\section{OBJETIVOS}

- Caracterizar a atuação educativa dos(as) enfermeiros(as) que implementam o programa de prevenção e controle das DSTs/AIDS nas unidades básicas de saúde.

- Identificar a percepção dos(as) enfermeiros(as) quanto às atividades educativas desenvolvidas na prevenção da AIDS;

- Identificar as atividades educativas na prevenção da AIDS, desenvolvidas pelos(as) enfermeiros(as) na rede básica municipal de saúde;

\footnotetext{
* Dissertação apresentada ao Curso de Mestrado em Enfermagem em Saúde Pública da UFPB, para obtenção do Grau de Mestre em Enfermagem. 1997

** Enfermeiro, Mestre em Saúde Pública/UFPB, Professor Assistente do Departamento de Enfermagem da UFRN e Doutoranto em Enfermagem Fundamental da Escola de Enfermagem de Ribeirão Preto da Universidade de São Paulo

***Enfermeira, Doutora em Enfermagem, Professora Adjunto do Departamento de Enfermagem da UFRN e Orientadora do trabalho
} 
-Descrever como são desenvolvidas as atividades educativas na prevenção da AIDS, pelos(as) enfermeiros(as), na rede básica municipal de saúde.

\section{REVISÃO DE LITERATURA}

Vários autores abordam as questões teóricas sobre educação em saúde em geral. A educação em saúde vem sendo entendida por muitos como uma maneira de fazer as pessoas do povo mudarem alguns comportamentos prejudiciais à saúde. Como exemplo, Marcondes apud PEREIRA (1983, p.10), descreve a educação em saúde como o "conjunto de atividades tendentes a influenciar ou modificar os conhecimentos, atitudes, crenças e o comportamento, visando à melhoria da saúde do indivíduo, da família e da comunidade".

Para outros autores, educar para a saúde é levar a população à compreensão e às soluções corretas que os profissionais conscientes, politizados e conhecedores da ciência já descobriram; ou seja, é conscientizar o povo que ainda não se conscientizou. Assim, só cabe entender a educação em saúde como uma educação baseada no diálogo e na troca de saberes (VASCONCELOS, 1989, p. 20).

Já FREIRE \& NOGUEIRA (1991, p. 65-66), fazendo a relação entre a educação em saúde e condições de vida apontam a educação popular como uma forma de ajudar as pessoas a transformarem e resolverem suas dificuldades.

Assim, a educação em saúde se constitui num aspecto da educação integral, envolvendo, portanto, o reconhecimento de que as ações educativas se processam em uma sociedade em mudança, como um processo contínuo. Busca-se não a mera adaptação do educando ao mundo já construído, mas alcançar, através desse processo, a própria mudança (DILLY \& JESUS, 1995, p. 101).

De acordo com Freire apud MONTEIRO (1995, p. 124) a educação se relaciona com o processo de conscientização, caracterizado pela ação crítica permanente do homem sobre a realidade.

Poucas abordagens teóricas em educação têm sido encontradas na literatura com relação à AIDS. Enquanto a educação em saúde, em geral, é enfocada na literatura de forma de transmissão de conhecimento, a educação em saúde em AIDS assume um caráter mais reflexivo e transformador nos trabalhos realizados recentemente.

Neste estudo, a educação em saúde está fundamentada na abordagem político-social, que, segundo SCHALL \& STRUCHINER (1995, p. 89), possibilita a participação efetiva das camadas socioeconômicas mais desfavorecidas, como sujeitos do processo político-cultural, e que se baseia nos trabalhos de Paulo Freire $(1975,1987)$, que propôs como tema central a humanização das relações humanas e a libertação do homem.

Segundo Homans e Agletton apud SCHALL \& STRUCHINER (1995, p. 96), os educadores comprometidos com a transformação social necessitam considerar: as mudanças no nível das idéias, separandose os argumentos científicos das considerações morais; as relações sociais entre educadores e educandos, com transformação das atitudes de discriminação; processos políticos e alocação de recursos, visando à organização de movimentos de reestruturação dos serviços de saúde e acesso de recursos para a saúde.

\section{CONSIDERAÇÕES METODOLÓGICAS}

Estudo do tipo exploratório descritivo, com abordagem qualitativa, objetivando analisar a participação dos enfermeiros na prevenção da AIDS ao nível da rede básica municipal de saúde de Natal - Rio Grande do Norte.

A população foi composta pelos 35 enfermeiros(as) que trabalhavam nas 10 UBSs que implementam o programa de prevenção e controle das DSTs/AIDS no município. Foram selecionadas 10 (dez) enfermeiras, uma por UBS, que desenvolviam atividades na prevenção da AIDS.

Foi utilizado um roteiro de entrevista e um gravador como recurso auxiliar, durante a realização da entrevista realizada nas UBSs, onde as enfermeiras atuavam. Para proceder a análise dos dados foi utilizado o método de análise de conteúdo, visando a detectar as temáticas expressas pelas entrevistadas com relação às suas ações educativas frente à AIDS.

\section{APRESENTAÇÃO, ANÁLISE E DISCUSSÃO DOS RESULTADOS}

As temáticas identificadas nos depoimentos, referentes ao questionamento abordado com as enfermeiras sobre seu entendimento da educação em saúde de uma forma geral, foram agrupadas e classificadas em 4 categorias: prevenção de doenças (4), repasse de informações (3), conscientização/cidadania (2) e treinamento/atualização (1).

A educação em saúde no entendimento dessas profissionais se resume basicamente em transmitir para a população informações, conhecimentos e práticas educativas consideradas saudáveis e que previnam as 
doenças. Ou seja, ensinar à população o que é correto para não adoecer.

... seriam palestras, seriam orientações, programas destinados a orientações de determinados assuntos, que orientem a saúde preventiva (...) tudo que você pudesse fazer para orientar essas pessoas em relação à saúde...

Constatamos, assim, que a maioria das pesquisadas (7), possuem entendimentos restritos e tradicionais da educação em saúde, refletindo, por conseguinte, diretamente nas suas ações educativas na comunidade, como verificamos nas discussões das categorias relacionadas à prevenção de doenças e no repasse de informações, que expressam uma significativa compreensão da educação em saúde embasada numa metodologia de transmissão de informações, com abordagem essencialmente comportamentalista.

Entretanto vale ressaltar que, em apenas 2 (dois) discursos, detectamos um entendimento bastante amplo da educação em saúde, como sendo um processo de capacitação e conscientização crítica dos indivíduos para lutarem pelos seus direitos sociais em relação à saúde e conquistarem uma melhor qualidade de vida através da transformação do seu próprio ambiente, ou seja, deixaram transparecer idéias de uma prática voltada para conquista da cidadania plena, como podemos observar no recorte a seguir.

... fazer com que essa população modifique o seu hábito de vida, transforme essa pessoa (...) que ela realmente passe a ver melhor seus direitos (...) é você conseguir transformar as pessoas (...) com base nessas informações, possa transformar o seu ambiente de vida ...

Essa perspectiva, corroborando os entendimentos das pesquisadas, DILLY \& JESUS (1995, p. 101), afirmam que a educação em saúde constitui um aspecto da educação integral, envolvendo, portanto, o reconhecimento de que as ações educativas se processam em uma sociedade em mudanças, como um processo contínuo. Busca-se não a mera adaptação do educando ao mundo já construído, mas alcançar, através desse processo, a própria mudança.

Com relação ao questionamento às enfermeiras sobre sua percepção em torno da educação em saúde na prevenção da AIDS no município, foram elaboradas 4 (quatro) categorias: ação insuficiente do programa (8), falta de estrutura do serviço e apoio institucional (7), atividades educativas do programa da AIDS (6) e comportamento inadequado dos clientes (6).

De acordo com os relatos, as enfermeiras expressaram a idéia de que o programa da AIDS, no município de Natal/RN, funciona de maneira insuficiente, com poucos resultados e, em alguns casos, não existe ação preventiva, como podemos verificar no recorte a seguir.

... eu considero praticamente inexistente educação em saúde na prevenção da AIDS. Eu vejo que não existe um programa de prevenção à AIDS tanto do ponto de vista do uso da prevenção através da camisinha, quanto a questão educativa ...

Podemos constatar que o programa de prevenção da AIDS do município de Natal, segundo percepção das pesquisadas, ainda está muito longe de atender às expectativas dos profissionais envolvidos na execução das atividades educativas e da população que deveria ser beneficiada com as ações de tal programa. As enfermeiras que relataram concretamente a ausência de estrutura no serviço básico de saúde, como também a falta de apoio e omissão institucional relacionado ao programa de prevenção da AIDS do município, colocando como pontos fundamentais a falta de infra-estrutura, recursos materiais e educativos, camisinhas, treinamentos e trabalho em equipe. Podemos verificar no relato a seguir.

... a Secretaria Municipal de Saúde e também a Estadual são omissas com relação a isso, não têm um trabalho ofensivo (...) não dão infra-estrutura, que é o acesso da população ao uso da camisinha e, segundo, não têm uma campanha educativa para atingir todos os grupos populacionais ...

As ações concretamente existentes no programa de prevenção da AIDS do município, desenvolvidas tanto nas unidades de saúde quanto nas comunidades, destacando-se as distribuições de camisinhas e panfletos, peças educativas, controles das DSTs/AIDS e palestras. Todavia, as atividades descritas pelos enfermeiros participantes do estudo podem ser classificadas como sendo limitadas e de pouca abrangência.

Neste sentido, as atividades educativas desenvolvidas pelas enfermeiras nas suas práticas cotidianas nos serviços básicos de saúde refletem que o impacto desse programa na unidade/comunidade deixa muito a desejar, e que precisam ser revistos urgentemente, todos esses fatores apontados e relacionados pelos pesquisados, para que o programa de prevenção da AIDS da Secretaria Municipal de Saúde possa funcionar adequadamente.

As pesquisadas expressaram em seus relatos idéias de inadequações comportamentais das pessoas que dificultam a educação em saúde na prevenção da AIDS. Colocaram principalmente a falta de uso da camisinha durante as relações sexuais, atribuindo-os a fatores como falta de informação, questão cultural, tabus sociais, promiscuidade e negação. O recorte a seguir explicitam melhor a situação acima descrita. 
... Já conheci várias pessoas que só estão usando a camisinha quando está havendo uma ejaculação. Vão prevenir a AIDS? (...) e só usam a camisinha assim ...

MONTEIRO (1995, p. 122-123), em seus estudos sobre os conhecimentos e a opinião dos jovens acerca de temas relacionados à epidemia de HIV/AIDS dentro da realidade brasileira, revelou que existe um preconceito dessa população em relação ao uso da camisinha, e que, embora o nível de informação sobre a transmissão e a prevenção do HIV seja satisfatório, ele não tem sido suficiente para gerar comportamentos preventivos.

Portanto não se pode pensar em prevenção coletiva da AIDS sem antes levar em consideração todos esses aspectos sócio-econômico-culturais e políticos envolvidos nessa questão.

Quanto as atividades educativas desenvolvidas na unidade/comunidade na prevenção da AIDS, foram formuladas 3 categorias: atividades em AIDS na unidade, na comunidade e atividades relacionadas à Secretaria Municipal de Saúde.

As ações educativas relatadas pelas pesquisadas, desenvolvidas na unidade foram distribuição de camisinha com orientação, palestra no corredor/sala de espera, demonstração do uso correto da camisinha, notificação das DSTs/AIDS, trabalho educativo com as profissionais do sexo, trabalho de orientação aos agentes comunitários e atividades relacionadas: planejamento familiar, crescimento e desenvolvimento (C D) e prénatal.

Vale ressaltar que a maioria dessas atividades mencionadas são desenvolvidas de forma esporádica, individual e exclusivamente com a demanda espontânea que procura o serviço para o atendimento. Ou seja, não existe programação específica com metas, objetivos, estratégias, etc. O recorte abaixo mostra melhor essas atividades.

... nesse programa são feitas as orientações
sobre as DSTs e AIDS (...) teve umas
palestras que a gente realizou este ano, mas
não foram muitas, aqui no próprio posto,
aqui na sala de espera (...) a clientela com
doenças sexualmente transmissíveis (...) é
encaminhada do clinico para cá, para a
gente orientar e fazer a notificação ...

Quanto à distribuição do preservativo nas UBSs pesquisadas, a sua entrega ocorre de forma esporádica, espontânea e individual. As orientações sobre o uso correto, são fornecidas pelos(as) enfermeiros(as) e assistentes sociais, de forma rápida e sem utilização de materiais educativos.

Com relação às palestras, que são realizadas nos corredores ou espaços existentes em frente às salas de atendimento, normalmente são desenvolvidas pelas enfermeiras sem uma programação prévia, esporádica e com carência de recursos materiais e audiovisuais. Apenas uma enfermeira relatou a participação de uma equipe multiprofissional no desenvolvimento dessa atividade.

... a gente faz um trabalhinho em relação ao grupo de espera, mas isso não é feito com freqüência (...) a gente aproveita aí e faz algumas orientações para esse grupo de espera (...) só conversando, sem nenhum recurso material ...

Com relação ao trabalho comunitário, 4 enfermeiras disseram que desenvolviam algumas atividades fora da unidade, muito embora de forma isolada, esporádica e sem planejamento e participação da equipe multiprofissional, como orientação de peça educativa, palestras em escolas, trabalho educativo com as profissionais do sexo nas casas de drinks e trabalho educativo na praia e restaurantes com distribuição de camisinha, panfletos e orientações.

Quanto às atividades relacionadas à AIDS desenvolvidas pela SMS, mais especificamente as implementadas pela coordenação do programa municipal de prevenção da AIDS, segundo relatos das pesquisadas, parecem ser bastante modestas, em relação ao que poderia ser desenvolvidos nas unidades básicas de saúde e comunidades, restringia-se ao treinamento de agentes comunitários de saúde.

Entretanto, em 2 (dois) depoimentos, as enfermeiras expressaram a existência de grupos de teatros de rua, formados por agentes comunitários de saúde, que são coordenados e orientados pelos enfermeiros das unidades. Essas atividades educativas são realizadas com certa freqüência na comunidade, principalmente em escolas e creches do município, onde fazem as dramatizações e orientações sobre a prevenção das DSTs/ AIDS, com a distribuição de preservativos para a platéia que assiste às apresentações, que são geralmente crianças e adolescentes jovens, como mostra o discurso abaixo.

... Quanto à peça (...) o grupo recebe chamadas basicamente de escolas e creches do município e estado (...) é mais dirigida a jovens e crianças (...) Esse grupo é composto exclusivamente de agentes comunitários, é coordenado por nós na questão de passar informação correta ...

No contexto da AIDS, todas as iniciativas no sentido de estimular a discussão e conscientização dos indivíduos a respeito da sua prevenção merecem ser incentivadas e apoiadas. Portanto as atividades educativas na prevenção da AIDS, que levam em consideração os aspectos sócio-culturais, como a cultura popular da população trabalhada e que utiliza formas criativas de abordar a temática da AIDS, podem desempenhar um 
papel importante na redução da disseminação desta enfermidade, como por exemplo, a peça teatral desenvolvida pelos agentes comunitários de saúde.

Portanto essa utilização de práticas educativas inovadoras na prevenção da AIDS merece um destaque considerável, necessitando, pois, de estudos que avaliem essas práticas populares como campo de investigação.

Quanto ao trabalho com grupos específicos, apenas 2 (duas) enfermeiras desenvolvem efetivamente uma ação educativa, uma com profissionais do sexo nas casas de "drinks". Esse trabalho tem a participação de outros profissionais da SMS, sendo realizado com freqüência nos prostíbulos. Já a outra atividade relatada é desenvolvida na comunidade direcionada à população da área de abrangência da unidade, constituindo um trabalho planejado, com utilização de recursos materiais e participação em conjunto com outros profissionais da unidade. $\mathrm{O}$ recorte abaixo exemplificam a situação apresentada.

... nós vamos as casas de drinks (...) expomos a palestra, também é participativo, a gente procura fazerem uma dinâmica (...) nós estamos fazendo diretamente com a psicóloga da secretaria e a assistente social (...) o objetivo é atingir todas as casas de drinks.

Nas UBSs pesquisadas, não se observou o trabalho multiprofissional e em conjunto, sugerindo o despreparo dos profissionais de saúde quanto ao trabalho coletivo em saúde, o que nos remete a refletir e questionar sobre a formação acadêmica desses profissionais, a quem eles estão servindo, bem como, sobre os próprios objetivos e finalidades dos serviços básicos de saúde.

Esses resultados apontam para uma maior reflexão e aprofundamento científico quanto à temática educativa em saúde, especificamente na prevenção da AIDS, no que se refere ao envolvimento da equipe multiprofissional nas ações educativas individuais e coletivas a serem desenvolvidas nas UBSs.

As temáticas identificadas nos relatos das pesquisadas, referentes ao questionamento abordado sobre as sugestões para a melhoria das atividades educativas na prevenção da AIDS, foram agrupadas e classificadas em 6 (seis) categorias: estruturação do serviço(10), preparo dos profissionais no programa da AIDS (8), promoção de trabalho em equipe (7), valorização do profissional (3) e promoção de trabalho na comunidade (2).

Segundo as opiniões das enfermeiras, seria necessária toda uma infra-estrutura que desse suporte para o funcionamento do programa, sendo que as mais citadas e fundamentais foram: disponibilidade de materiais educativos (panfletos, álbuns seriados); camisinhas em quantidade suficiente para serem distribuídas com a demanda espontânea e comunidade; recursos audiovisuais, recursos humanos para trabalhar com a AIDS; espaço físico adequado e principalmente apoio da Secretaria Municipal de Saúde oferecendo todas essas necessidades básicas. Para um melhor entendimento da situação apresentada, seguem-se um recorte dos discursos das enfermeiras a respeito desse enfoque.

... para mim infra-estrutura é isso; é a camisinha, é material educativo de campanha publicitária que seja enfática (...) para trabalho em grupo, material audiovisual para se trabalhar a parte educativa (...) as unidades não têm espaço para trabalho de grupo....

Diante do exposto, constatamos que as enfermeiras pesquisadas possuem, de uma maneira geral, um bom senso crítico quanto às necessidades de melhorias nos serviços básicos de saúde, não só com relação às atividades em AIDS. Apontaram a necessidade urgente de uma infra-estrutura que seja capaz de atender às expectativas dos serviços quanto às ações a serem desenvolvidas, e da própria população quanto à minimização dos seus problemas de saúde.

Com relação aos treinamentos, 8 (oito) enfermeiras, relacionados à falta de preparo das mesmas para trabalhar no programa de prevenção da AIDS, foram unânimes em relatarem a necessidade urgente de treinamentos e atualizações sobre AIDS, com vistas a desenvolverem com mais segurança o trabalho educativo na prevenção dessa enfermidade. O recorte a seguir reflete melhor a situação em questão.

... os profissionais de saúde estão extremamente despreparados também, não só pela questão técnica da prevenção da AIDS (...) quanto à metodologia de trabalho com a população, porque a gente ainda é muito tradicional na forma de passar informações...

Diante disso, Meneghin apud DRESLER \& BOEMER (1991, p. 72) referem que é importante que o enfermeiro esteja atualizado, acompanhe o desenvolvimento das pesquisas e dessa forma se conscientize sobre suas possibilidades para atender o cliente. Referem-se também, à necessidade de conhecimentos sobre a sexualidade humana e à realidade da morte iminente, geralmente cercados de tabus e preconceitos, quando se dispõem a prestar assistência.

Vale ressaltar que esse engajamento de toda a equipe multiprofissional depende, principalmente, do estímulo e motivação de cada profissional em trabalhar no programa, como também das condições de vida e trabalhos a que estão submetidos os mesmos.

Portanto a educação em saúde comprometida com a transformação social dos indivíduos envolvidos 
nesse processo educativo, tornando-os cidadãos, necessita ser estimulada como prática coerente, contínua e sensibilizada com o desenvolvimento social e político da população. Sendo assim, todo os profissionais, não só os enfermeiros, podem desenvolver ações educativas em saúde em todo e qualquer contato com a população, visando em última analise, à melhoria das condições e qualidade de vida da população.

\section{CONSIDERAÇÕES RECOMENDAÇÕES}

\section{FINAIS}

$\mathbf{E}$

As pesquisadas demonstraram um entendimento pouco diversificado, restrito, tradicional e formal da educação em saúde, com uma visão comportamentalista e abordagem de repasse de informações.

O programa possui ações insuficientes e não vêm atendendo as expectativas dos profissionais envolvidos, as atividades são pouco abrangentes, sem objetividade, de natureza acríticas e não conduzem as mudanças necessárias. As ações educativas desenvolvidas pelas pesquisadas tem como características a falta de planejamento, não envolvimento da equipe, atividades isoladas e esporádicas, com utilização de abordagens comportamentalista e inexistência de um planejamento específico do programa.

Quanto as sugestões apontadas, demonstraram um senso crítico e sensibilização quanto à prevenção da AIDS, expressaram a necessidade urgente de: estruturação dos serviço de saúde, envolvimento da equipe, capacitação dos profissionais e alocação de recursos financeiros e materiais.

Diante dos achados torna-se necessária uma reavaliação conjunta dos objetivos, metas, estratégias e ações educativas a serem implementadas, capacitação dos recursos humanos envolvidos na operacionalização do programa e a alocação de recursos materiais necessários. Bem como, promover discussões ampla sobre o programa envolvendo os profissionais, comunidade e coordenação central.

Novas investigações sobre a temática sejam desenvolvidas, envolvendo também discussão interinstitucional entre os serviço de saúde e os órgãos formadores dos profissionais área, sobre o processo educativo em saúde. Portanto, alguns questionamentos a serem pesquisados, entre outros, seriam: Que estratégias de educação em saúde são mais efetivas na conscientização da população na prevenção da AIDS? Quais as conseqüências da educação preventiva em AIDS promovida pela equipe multidisciplinar?

\section{EDUCATIONAL ACTIVITIES ON AIDS PREVENTION IN A MUNICIPAL BASIC HEALTH UNIT: NURSE PARTICIPATION}

This is an exploratory descriptive study with a qualitative approach that had as its purpose to characterize the educational activities of the nurses that work in the prevention and control program for sexually transmittable diseases and AIDS in the municipality of Natal, RN. The study was conducted in the basic health care units with 10 (ten) nurses that implemented educational activities in AIDS prevention. A structured interview was used and content analysis techniques were applied to the data. It was identified that the majority of the nurses understood health education as the transmission of information and that the AIDS prevention program had insufficient actions, lacked structure in the service settings and institutional support by the Municipal Secretariat of Health. The program activities were characterized as traditional limited, sporadic, and lacking human and material resources.

KEY WORDS: nurse, education health, AIDS prevention

\section{ACTIVIDADES EDUCATIVAS EN LA PREVENCIÓN DEL SIDA EN UNA RED BÁSICA MUNICIPAL DE SALUD: PARTICIPACIÓN DEL ENFERMERO}

Este es un estudio exploratorio descriptivo, con una abordaje cualitativa, que tuvo como objetivo caracterizar la actuación educativa de los enfermeros que implementan el programa de prevención y control de las ETS y SIDA en la ciudad de Natal (RN). Fue realizado en las unidades básicas de salud, con 10 (diez) enfermeros que actuaban desarrollando acciones educativas en la prevención del SIDA. Se utilizó una guía de entrevista estructurada. Para el análisis de los datos se utilizó la técnica de análisis de contenido. Se identificó que la mayoría de los enfermeros entendieron la educación en salud como un repaso de las informaciones, el programa de prevención del SIDA tenía una acción insuficiente, con ausencia de estructura en el servicio y omisión de la Secretaria Municipal de Salud, en lo que se refiere a apoyo institucional. Las actividades en el programa eran tradicionales, poco amplias, esporádicas y sin recursos materiales y humanos capacitados. 


\section{REFERÊNCIAS BIBLIOGRÁFICAS}

01. DILLY, C.M.L.; JESUS, M.C.P.de. Processo educativo em enfermagem: das concepções pedagógicas à prática profissional. São Paulo: Robe, 1995. 190 p.

02. DRESLER, D.; BOEMER, M.R. O significado do cuidado do paciente com AIDS uma perspectiva de compreensão. Rev.Bras.Enfermagem, Brasília, v. 41, n. 1, p. 70-81, jan./mar. 1991.

03. FREIRE, P.; NOGUEIRA, A. Que fazer: teoria e prática em educação popular. 3. ed. Petrópolis: Vozes, 1991. $71 \mathrm{p}$.
04. MONTEIRO, S. Projeto viva a vida: prevenindo a AIDS na escola. In: CZERESNIA, D. et al. AIDS: pesquisa social e educação. São Paulo: Hucitec, 1995. Cap. 6, p. 122-136.

05. PEREIRA, I.M.T.B. Educação em saúde em serviços de saúde. São Paulo, 1983. p. 8-21. Dissertação (Mestrado em Enfermagem) - Escola Paulista de Medicina.

06. SCHALL, V.T.; STRUCHINER, M. Educação no contexto da epidemia de HIV/AIDS: teorias e tendências pedagógicas. In: CZERESNIA, D. et al. AIDS: pesquisa social e educação. São Paulo: Hucitec,1995. Cap. 5, p. 84-105.

07. VASCONCELOS, E.M. Educação popular nos serviços de saúde. São Paulo: Hucitec, 1989. $139 \mathrm{p}$. 